\title{
Tratamiento farmacológico o quirúrgico del paciente con sobrepeso u obesidad
}

\author{
Fernando Carrasco ${ }^{1}$, Mónica Manrique ${ }^{2}$, \\ María Pía de la Maza ${ }^{3}$, Manuel Moreno ${ }^{4}$, Cecilia Albala², \\ Jaime G arcía ${ }^{5}$, Jaime Díaz ${ }^{6}$, Claudio Liberman ${ }^{7}$. \\ Statement about the medical \\ and surgical treatment \\ of overweight and obesity
}

\begin{abstract}
This is an updated review of the available treatments for obesity, that can be used when lifestyles modifications fail. Using the available information and the experience of the members of this advisory group, a recommendation is given about the most useful treatments, according to the severity of obesity and its complications. With regards to pharmacological treatments, only sibutramine and orlistat are approved on a worldwide basis for the treatment of obesity. These medications achieve a $10 \%$ higher weight reduction than lifestyles modification. A third medication, rimonobant, is also more effective than lifestyles modifications, but it was withdrawn due to psychological safety issues. The indications for surgical treatment and a brief description of the available techniques, success rates and complications are outlined. Finally, the need to have follow up protocols for patients and the formation of multidisciplinary treatment teams is underscored (Rev Méd Chile 2009; 137: 972-81).
\end{abstract}

(Key w ords: Anti-obesity agents; Bariatric surgery; Obesity)

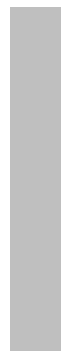

\begin{abstract}
Recibido el 8 de marzo, 2009. Aceptado el 18 de marzo, 2009.
Documento de consenso del Grupo de Estudio para la Obesidad, de la Sociedad Médica de Santiago.

${ }^{1}$ Departamento de Nutrición, Facultad de Medicina, Universidad de Chile, Santiago, Chile. 2Instituto Médico Nutramed, Santiago, Chile. ${ }^{3}$ Instituto de Nutrición y Tecnología de los Alimentos, Universidad de Chile, Santiago, Chile. ${ }^{4}$ Departamento de Nutrición, Diabetes y Metabolismo, Pontificia Universidad Católica de Chile, Santiago, Chile. ${ }^{5}$ Centro Médico CAPREDENA, Santiago, Chile. ${ }^{6}$ Unidad de Diabetes, Hospital San Juan de Dios, Santiago, Chile. ${ }^{7}$ Departamento de Endocrinología, Hospital Clínico de la Universidad de Chile, Santiago, Chile.
\end{abstract}

A demás de las medidas no farmacológicas, el uso de medicamentos puede ser de utilidad en algunos pacientes con sobrepeso u obesidad.

Correspondencia a: Dr. Fernando Carrasco N. Departamento de Nutrición, Facultad de Medicina, Universidad de Chile. Independencia 1027, Independencia, Santiago. Fono: 7586954. Fax: 7378778. E mail: fcarrasc@med.uchile.cl
Según consenso internacional, se justifica el uso de fármacos en obesidad cuando fracasa el tratamiento con dieta, ejercicio y manejo conductual en pacientes con IMC $>30 \mathrm{~kg} / \mathrm{m}^{2}$ o con IMC $>27 \mathrm{~kg} /$ $\mathrm{m}^{2}$ y comorbilidades de relevancia médica (diabetes tipo 2, hipertensión arterial, dislipidemia, artropatía, etc. $)^{1}$.

En la indicación de un tratamiento farmacológico en obesos se deben considerar los factores etiológi- 
cos, la respuesta terapéutica (efectos farmacológicos y colaterales), dosis apropiadas, interacciones farmacológicas, contraindicaciones médicas o psiquiátricas. El uso de fármacos en la actualidad sólo se justifica en el contexto de un tratamiento integral (dieta, actividad física, terapia conductual). Debe reservarse para adultos y excepcionalmente se pueden utilizar en adolescentes. La obesidad es una patología crónica, por lo que al iniciar una terapia farmacológica, se debe tener en cuenta que el medicamento haya demostrado su eficacia a largo plazo (mínimo 2 años) y que el riesgo asociado al medicamento sea inferior al de la obesidad y sus complicaciones. La Tabla 1 muestra las caracteństicas ideales que la OMS ha señalado para un fármaco para el tratamiento de pacientes obesos ${ }^{2}$.

Los fármacos que se han utilizado en el tratamiento de la obesidad actúan a través de 3 mecanismos principales:

1. Disminuyendo la ingesta calórica por inhibición del apetito (anorexígenos) o aumento de saciedad (sacietógenos).
2. Aumentando el gasto energético y la oxidación de lípidos (termogénicos).

3. Inhibiendo la digestión y absorción de macronutrientes de la dieta (inhibidores de enzimas digestivas).

La Tabla 2 resume los fármacos en uso en la actualidad para ayudar al manejo de la obesidad, de acuerdo a su mecanismo de acción.

\section{FÁRMACOS QUE HAN SIDO APROBADOS PARA EL TRATAMIENTO DE LA OBESIDAD}

En la actualidad solamente sibutramina y orlistat, han sido aprobados a nivel internacional para su uso a largo plazo en el manejo de la obesidad, ya que cuentan con estudios clínicos que demuestran su eficacia y seguridad. Rimonabant fue aprobado en nuestro país y en algunos países europeos, aunque la FDA (Food and Drugs Administration), nunca permitió su venta en Estados Unidos de

Tabla 1. Características ideales de un fármaco para el tratamiento de la obesidad según la 0 M S

- Reducción demostrada en peso y enfermedades asociadas

- Efectos secundarios tolerables o transitorios

- Sin reacciones adversas mayores después de años de uso

- Eficacia mantenida a largo plazo

- Sin propiedades adictivas

- Mecanismo (s) de acción conocido (s)

- Costo razonable

OMS: Organización Mundial de la Salud².

Tabla 2. Clasificación de los fármacos utilizados para el tratamiento de la obesidad según su mecanismo de acción

Inhibidores del apetito o estimulantes de saciedad:

- adrenérgicos controlados*: dietilpropión, mazindol, fentermina

- adrenérgicos no controlados: efedrina

- inhibidores selectivos de recaptura de serotonina: fluoxetina, sertralina

- acción dual (adrenérgico-serotoninérgico): sibutramina

- inhibidores del receptor 1 de endocanabinoides: rimonabant

Termogénicos-lipolíticos: efedrina/cafeína

Inhibidor de absorción de grasas: orlistat

*Controlados por el Instituto de Salud Pública y Ministerio de Salud 
Norteamérica. Recientemente la Agencia Europea de Medicamentos prohibió su comercialización en Europa y el laboratorio farmacéutico decidió retirarlo del mercado en todo el mundo.

Sibutramina. Este medicamento presenta un mecanismo de acción doble, al inhibir la recaptura sináptica de noradrenalina y serotonina. Al ser ingerido por vía oral produce una disminución de la ingesta de alimentos dosis-dependiente. La dosis habitual es de 10-15 mg/día. Presenta un efecto prolongado asociado a la vida media de sus metabolitos, por lo cual debe indicarse en dosis única matinal. La estimulación del sistema adrenérgico induce un leve efecto termogénico, con un aumento de hasta 5\% en el gasto energético de reposo y un menor descenso del metabolismo en la fase de reducción de peso.

Se han publicado alrededor de 10 estudios prospectivos, aleatorios y controlados que demuestran su eficacia. Uno de éstos es el Multicenter European Sibutramine Trial of Obesity Reduction and Maintenance Trial (STORM) ${ }^{3}$, que demostró que los pacientes tratados con el fármaco por un período de 2 años perdieron $10,2 \mathrm{~kg}$ en comparación con $4,7 \mathrm{~kg}$ en el grupo placebo, comprobando la eficacia de sibutramina en la reducción y mantención del peso. Los efectos colaterales más frecuentes son sequedad bucal, constipación, cefalea, insomnio, astenia, aumento de presión arterial y frecuencia cardíaca. Su uso está contraindicado en HTA no controlada, cardiopatía coronaria, insuficiencia cardíaca, arritmias, accidente cerebrovascular y en pacientes en tratamiento con inhibidores de la monoaminooxidasa. Por el riesgo de inducir síndrome serotoninérgico se debe tener precaución con el uso concomitante de antidepresivos inhibidores de la recaptura de serotonina (fluoxetina, sertralina) y antijaquecosos (ergotamina, sumatriptan).

Al iniciar el uso de sibutramina debe controlarse al paciente a las 2 semanas de tratamiento, para evaluar su tolerancia, con especial énfasis en la evolución de la presión arterial. En un metaanálisis se encontró que $1,1 \%$ de los pacientes tratados con sibutramina contra $0,6 \%$ de los pacientes con placebo, debieron ser retirados del estudio debido a un aumento de su presión arterial $^{4}$. Se ha observado que una disminución de 5\%-10\% del peso corporal se asocia a una mejoría en los factores de riesgo asociados al sobrepeso; los pacientes tratados con sibutramina muestran una leve mejoría del colesterol HDL, niveles de triglicéridos, ácido úrico, circunferencia de cintura y mejoría en el control glicémico en pacientes diabéticos ${ }^{5}$.

Orlistat. Este fármaco es un inhibidor de la lipasa pancreática, que al disminuir la hidrólisis intestinal de los triglicéridos provenientes de la dieta, impide la absorción de alrededor de $30 \%$ de ellos. En ensayos clínicos en humanos obesos se ha mostrado un efecto reductor del peso significativamente mayor que con placebo, y una mayor proporción de pacientes que mantienen una reducción de $5 \%$ y $10 \%$ del peso inicial, después de 2 años de seguimiento ${ }^{6}$. Los estudios de dosis-respuesta han determinado que el efecto máximo, medido por la excreción de grasa fecal, se observa al usar una dosis diaria de $360 \mathrm{mg}$ (120 mg tres veces al día, con cada comida $)^{7}$. Los resultados de varios estudios clínicos muestran un promedio de disminución de peso entre $5 \%$ y $10 \%$ del peso inicial contra $2 \%$ a $6 \%$ en el grupo placebo ${ }^{8}$. Torgerson y cols, en más de 3.000 sujetos, demostró que el tratamiento con orlistat más intervención en el estilo de vida disminuyó el peso en $11 \%$ y $7 \%$ a un año y 4 años, respectivamente, comparado con $6 \%$ y $4 \%$ en el grupo placebo, más cambio de estilo de vida ${ }^{9}$. Los efectos adversos se relacionan con su mecanismo de acción, por cuanto frecuentemente los pacientes presentan deposiciones blandas y aceitosas. Aproximadamente $75 \%$ de los pacientes tratados con orlistat presentan uno 0 más efectos colaterales de tipo gastrointestinal ${ }^{8}$. En menos de $5 \%$ de los casos puede aparecer esteatorea franca, diarrea, urgencia e incontinencia fecal. En estas tres situaciones se debe suspender el fármaco. Además de la reducción de peso, con orlistat se ha estudiado su efectividad en mejorar el control de las comorbilidades, demostrándose que, asociado a cambios en el estilo de vida, previene en 37\% la aparición de diabetes en un plazo de 4 años, además de reducir las dosis de hipoglicemiantes orales, mejorar el control glicémico y aumentar la sensibilidad insulínica ${ }^{9}$. También se han obtenido bajas significativas de los niveles plasmáticos de colesterol total y colesterol $\mathrm{LDL}^{10}$. En un estudio multicéntrico europeo se observó una reducción significativa en la presión arterial diastólica en comparación con placebo ${ }^{11}$. 
Otro inconveniente es que puede provocar malabsorción de vitaminas liposolubles, siendo recomendable, ante su uso prolongado, la suplementación oral de vitaminas A, D y E. Está contraindicado el uso de orlistat en síndromes de malabsorción crónica, colestasis y trastornos digestivos agudos.

Rimonabant. Este fármaco actúa en el sistema endocanabinoide, sistema fisiológico relacionado con las sensaciones placenteras a través de la vía mesolímbica dopaminérgica, que está constituido por receptores CB1 y CB2, y sus ligandos endógenos, la anadamida y el 2-araquidonoilglicerol. Los receptores CB1 se encuentran en el cerebro en las áreas relacionadas con la alimentación, en las células del tejido adiposo, en el tracto gastrointestinal y en el hígado, en cambio los receptores CB2 se encuentran principalmente en células del sistema inmune. El uso de rimonabant, un inhibidor selectivo del receptor CB1, demostró en estudios controlados en voluntarios humanos una mayor reducción del peso corporal en los pacientes tratados que en el grupo control, con mayor descenso en los triglicéridos y mayor incremento en los niveles de colesterol HDL ${ }^{12}$. Aunque los efectos colaterales observados con el fármaco eran poco frecuentes, su principal limitación fue el riesgo de inducir fenómenos depresivos y potencialidad de suicidio, por lo cual no recibió la aprobación de la FDA. Aun cuando en Europa y en muchos países, incluyendo Chile, fue aprobado su uso con la recomendación de no indicarlo a pacientes con antecedentes de depresión o en tratamiento por trastornos del ánimo, una reciente revisión de la Agencia Europea de Medicamentos (EMEA) recomendó en octubre de 2008 suspender su comercialización en Europa, ante lo cual el laboratorio fabricante decidió suspender la comercialización y los ensayos clínicos en todo el mundo hasta un adecuado análisis del perfil de riesgo-beneficio.

\section{FÁrMACOS APROBADOS PARA OTRAS INDICACIONES Y QUE PRODUCEN BAJA DE PESO MENOR A 5\% DEL PESO CORPORAL INICIAL}

Fluoxetina. Existe una frecuente asociación entre cuadros depresivos que cursan con angustia 0 ansiedad, y ganancia de peso por sobreingesta alimentaria. Se ha observado que los antidepresivos tricíclicos inducen un incremento del apetito, a diferencia de la fluoxetina, que promueve una reducción del peso por su acción serotoninérgica (inhibición de la recaptura de la serotonina). Estudios en pacientes obesos sin depresión, han mostrado un efecto dosis-dependiente del fármaco sobre el apetito (20-80 mg/día), con especial acción en pacientes con ingesta compulsiva (trastorno por atracones o binge-eating disorder), siendo también efectiva para reducir la ingesta en pacientes con estrés o ansiedad, 0 en quienes suspenden el hábito tabáquico. Un metaanálisis de 11 estudios sobre el efecto de fluoxetina administrada durante 28 semanas demostró una baja promedio de $3,3 \mathrm{~kg}$ de peso corporal ${ }^{13}$. A pesar de que la fluoxetina puede ser utilizada por períodos prolongados, se ha demostrado que su efectividad en el control del peso corporal disminuye progresivamente después de los 6 meses de tratamiento ${ }^{14}$.

Sertralina. Este antidepresivo inhibidor de la recaptura de serotonina, en algunos pacientes produce un efecto ansiolítico y aumento en la sensación de saciedad al utilizarse en dosis de 50 a $100 \mathrm{mg} /$ día. $\mathrm{Si}$ bien su efecto sobre la ingesta de alimentos es menos marcado que el de la fluoxetina, también es menor la frecuencia de efectos colaterales ${ }^{15}$.

Metformina. Este fármaco, sensibilizador de la acción insulínica, puede usarse en obesos con resistencia a la insulina, por sus acciones inhibitorias de la neoglucogénesis hepática, disminución de la absorción de glucosa a nivel del tracto intestinal y estimulante de la acción de la insulina a nivel celular. En algunos pacientes el fármaco favorece la baja de peso a través de sus efectos metabólicos. El mejor estudio para evaluar el efecto de metformina sobre el peso fue el Programa de Prevención de Diabetes realizado en individuos con intolerancia a la glucosa, mayores de 25 años e IMC mayor a $24 \mathrm{~kg} / \mathrm{m}^{2}{ }^{16}$. No se clasifica como una droga para bajar de peso ya que los resultados de los tratamientos con metformina, muestran una disminución menor a $5 \%$ del peso corporal inicial.

Bupropion. Es un fármaco antidepresivo inhibidor de la recaptura de norepinefrina y dopamina, de 
utilidad en el tratamiento para la suspensión del tabaco. Existen varios estudios que demuestran una disminución del peso en pacientes tratados, con o sin depresión. El efecto en el peso se observó con altas dosis de 300-400 mg diarios lo que hace aumentar el riesgo de eventos adversos como, por ejemplo, hipertensión arterial severa ${ }^{17}$.

Topiramato. Es un agente antiepiléptico que modula los canales de calcio y sodio además de bloquear los receptores de glutamato. Se ha demostrado su eficacia en el tratamiento de la obesidad, así como también efectos favorables en las comorbilidades asociadas al exceso de peso. También se ha visto mejoría de la sintomatología en patologías de la conducta alimentaria tales como el trastorno por atracones (binge eating disorder) y comer nocturno (night eating syndrome), aunque son estudios pequeños y de corta duración. Las dosis utilizadas van desde los 25 a $400 \mathrm{mg}$ diarios, en una o dos tomas al día; se recomienda comenzar el tratamiento con dosis bajas e incrementarla semanalmente en forma gradual. Los efectos adversos observados fueron parestesias, somnolencia, dificultad en la concentración, memoria y atención ${ }^{18}$.

Análogos del GLP-1 (Exenatide). El GLP-1 o enteroglucagón es una proteína derivada del proglucagón y secretada por las células $\mathrm{L}$ del íleon terminal en respuesta a la ingesta alimentaria; es una hormona insulinotrópica (incretina), favorece la sensibilidad a la insulina, protege a la célula beta, disminuye la secreción de glucagón y estimula la secreción de insulina, pero adicionalmente tiene un efecto sacietógeno central. Se estudió el efecto de Exendin-4, $10 \mu \mathrm{g}$ subcutáneo al día versus placebo, en pacientes diabéticos con fracaso de tratamiento a dosis máximas de sulfonilureas, observándose mejor control metabólico y disminución promedio de 1,6 kg ${ }^{19}$.

Pramlintida. Es un análogo sintético de la amilina (péptido secretado por las células beta pancreáticas), aprobado por la FDA para el tratamiento de la diabetes. En un estudio en pacientes con diabetes tipo 2 tratados con insulina, se les administró pramlintida subcutánea 2 ó 3 veces al día y se observó una disminución de 2,6 kg en el peso corporal y una disminución en la hemoglo- bina glicosilada de $0,5 \%$. El efecto adverso más frecuente fue náuseas, el que fue leve y ocurrió mayoritariamente en las primeras 4 semanas de tratamiento 20 .

\section{FÁRMACOS EN ESTUDIO \\ PARA EL TRATAMIENTO DE LA OBESIDAD}

Leptina. Hormona inductora de saciedad, secretada por los adipocitos. Su descubrimiento generó grandes expectativas para el tratamiento de la obesidad. Sin embargo ha demostrado ser útil sólo en el tratamiento de pacientes obesos con mutación del gen involucrado en su síntesis, condición rarísima en el ser humano, y muy baja eficacia en individuos con resistencia a la leptina, que constituyen una gran parte de los pacientes obesos, pero podría tener un rol en la mantención del peso perdido ${ }^{21}$.

Lorcaserina. Es un agonista serotoninérgico con una afinidad hasta 100 veces por el receptor 2 C, que se distribuye en el sistema nervioso central, especialmente en áreas relacionadas con la regulación del apetito, que sobre receptores periféricos involucrados en el daño de válvulas cardiacas asociado a uso de serotoninérgicos menos específicos como anorexígenos retirados del mercado (fenfluramina y dex-fenfluramina), o aquellos usados como antijaquecosos (ergotamina y sumatrip$\tan )^{22}$.

En el último tiempo se ha planteado el uso de terapia combinada de diferentes fármacos, de las cuales ya han sido patentadas fentermina-topiramato $\left(\mathrm{Q}\right.$ nexa $\left.{ }^{\circledR}\right)$, bupropión-naltrexona (Contra$\left.\mathrm{ve}^{\circledR}\right)$ y bupropion-zonisamida $\quad\left(\right.$ Empatic $\left.^{\circledR}\right)$. El concepto es usar fármacos con diferente mecanismo de acción para maximizar la reducción del peso, reduciendo el riesgo de efectos colaterales ocasionado por mayores dosis de cada uno de los fármacos usados como monoterapia ${ }^{23}$.

\section{FÁRMACOS CUYO USO ES DE ALTO RIESGO}

Las anfetaminas y la metanfetamina producen rápida dependencia y tolerancia, excitabilidad, irritabilidad y aumentan el riesgo cardiovascular (hipertensión arterial, arritmias). Además presen- 
tan un alto potencial de abuso (adicción). La fenilpropanolamina fue retirada del mercado mundial por su asociación con accidente cerebrovascular hemorrágico en mujeres ${ }^{25}$. Los derivados anfetamínicos tales como anfepramona (dietilpropión), fenproporex y mazindol, mantienen su aprobación por la FDA, y también por las autoridades sanitarias en Chile. Sin embargo, nuestro grupo no recomienda su uso por el alto riesgo de dependencia, la elevada frecuencia de efectos colaterales y la ineficacia sobre el control del peso a largo plazo ${ }^{24}$. Las hormonas tiroideas y los extractos de tiroides promueven pérdida de peso con gran compromiso del compartimiento muscular, aceleran la pérdida de masa ósea y se asocian a arritmias e hipertrofia cardiaca cuando se utilizan por períodos prolongados. El tiratricol o ácido triiodotiroacético también ha sido prohibido para su uso en obesidad considerando su potencial de riesgo como derivado de hormona tiroidea y sus efectos negativos en el eje hipofisiario tiroideo. Los diuréticos y laxantes inducen una reducción brusca de peso por pérdida de agua (deshidratación), con riesgo de alteraciones hidroelectrolíticas severas, como depleción de sodio, potasio, fosfato y magnesio.

\section{FÁRMACOS NO APROBADOS} PARA EL TRATAMIENTO DE LA OBESIDAD

La efedrina es un fármaco que estimula los receptores $\alpha 1, ß 1, ß 2$ y 33 -adrenérgicos, lo cual conduce a un incremento en el gasto energético y utilización de grasas como sustrato energético. La cafeína es una xantina que inhibe los receptores de adenosina y la fosfodiesterasa, demostrando un efecto sinérgico al asociarse con efedrina. Si bien en Chile la efedrina se encuentra incorporada en el registro sanitario, su uso para el tratamiento de la obesidad no está aprobado, aunque la combinación de efedrina-cafeína está patentada en algunos países europeos (Letigen ${ }^{\circledR}$ ), para el tratamiento de la obesidad ${ }^{26}$. Por su acción adrenérgica debe tenerse especial cuidado con su uso en pacientes con HTA, arritmia, antecedentes de accidente cerebrovascular, enfermedad coronaria y patologías psiquiátricas (cuadros ansiosos, crisis de pánico, sicopatías).

\section{FÁRMACOS SIN FUNDAMENTO CIENTÍFICO}

En los últimos años se han comercializado diversos productos naturales con supuesto efecto termogénico o lipolítico, tales como picolinato de cromo, piruvato de calcio, ácido hidroxicítrico, ephedra y L-carnitina. Estos productos naturales no han demostrado eficacia en estudios controlados realizados en humanos, e incluso se han reportado eventos cardiovasculares severos. Tampoco hay evidencia científica que apoye el uso de productos como spirulina, vinagre de manzana, ácido linoleico y centella asiática, entre otros compuestos. Un producto denominado "Chitosan", no ha mostrado diferencia en comparación con placebo en reducir la absorción de la grasa ingerida, en estudios controlados en humanos ${ }^{27}$, como tampoco han mostrado efectividad en el manejo de la obesidad substancias o fármacos que inhiben la absorción de hidratos de carbono como el inhibidor de alfa glucosidasa intestinal (acarbosa), o el inhibidor de la amilasa pancreática (faseolamina) ${ }^{28}$. En relación a los suplementos de fibra dietética, no existen estudios controlados que apoyen el uso de fibra dietaria como una alternativa eficaz en el tratamiento de la obesidad. Por lo tanto, no es recomendable el uso de fibras como metilcelulosa, glucomanan, goma guar, entre otras.

Nuestro Grupo de Estudio de la Obesidad considera que el uso de fármacos en el tratamiento de la obesidad debe ser cuidadoso, monitorizando la aparición de reacciones adversas, interacciones con otros medicamentos o agravación de comorbilidades. Por otra parte constituyen una valiosa herramienta terapéutica siempre y cuando sean usados en el contexto de un tratamiento integral, que implique terapia nutricional, psicológica y actividad física. Finalmente, considerando que la obesidad es una patología crónica, la terapia farmacológica debiera planificarse para el largo plazo.

\section{TRATAMIENTO QUIRÚRGICO DE LA OBESIDAD}

Los tratamientos que incluyen las medidas más conservadoras señaladas previamente, deben considerarse inicialmente como el enfrentamiento de elección. Sin embargo, en pacientes con obesidad 
severa y mórbida, una pérdida significativa de peso y mantenida en el largo plazo sólo se observa en alrededor de $5 \%$ de los pacientes ${ }^{29}$ por lo que se considera a la cirugía bariátrica como el método más efectivo para lograr ese objetivo. Dos metaanálisis han documentado la eficacia de distintos tipos de cirugía bariátrica, no sólo sobre la reducción del peso, sino también en la mejoría en los indicadores de riesgo asociados ${ }^{30,31}$.

Indicaciones de cirugía en obesidad. La indicación de cirugía para tratar a pacientes obesos se ha definido en consensos intemacionales y nacionales $2,32,33$. Se consideran elegibles para este procedimiento a pacientes con IMC igual o mayor de $40 \mathrm{~kg} / \mathrm{m}^{2}$, o con IMC igual o mayor de $35 \mathrm{~kg} / \mathrm{m}^{2}$ asociado a condiciones médicas relevantes como diabetes tipo 2 , hipertensión arterial, dislipidemias, cardiopatía comnaria, artropatía de grandes articulaciones o apnea obstructiva del sueño, entre otras condiciones. De igual forma se han señalado como contraindicaciones absolutas algunas patologías psiquiátricas (esquizofrenia, trastomos severos de la personalidad, depresión no controlada), adicción a fámacos o alcohol y antecedentes de mala adherencia y mal cumplimiento de tratamientos médicos ${ }^{33}$.

Tipos de técnicas quirúrgicas en obesidad. Existen varias modalidades de cirugía bariátrica, algunas sólo restringen la capacidad gástrica y otras que se combinan con un procedimiento de malabsorción. Las técnicas más ampliamente difundidas y aplicadas en la actualidad son la gastroplastia con bypass gastro-yeyunal en $\mathrm{Y}$ de Roux abierto 0 laparóscopico (BPG), la banda gástrica ajustable laparoscópica, y de más reciente introducción, la gastrectomía vertical en manga $(\mathrm{GVM})^{34-36}$. Las técnicas que sólo restringen la capacidad gástrica, como la gastroplastia con banda vertical y el banding gástrico han mostrado reducciones de peso menos significativas que el bypass gástrico ${ }^{37}$, mayor frecuencia de reoperaciones y conversiones a técnicas más complejas como el bypass gástrico y mayor recuperación del peso perdido a largo plazo, aunque aún se consideran atractivas por su menor complejidad técnica y su baja morbilidad postoperatoria.

El bypass gástrico es la técnica más recomendada en la actualidad $30,36,37$, siendo también la de mayor uso en Chile ${ }^{38-40}$. Este procedimiento ha mostrado inducir y mantener una pérdida satisfactoria de peso ${ }^{35,37,41}$ y en estudios nacionales se han reportado altas tasas de éxito ${ }^{38-40}$. La técnica consiste en la fabricación de un reservorio gástrico proximal (aproximadamente 15 a $30 \mathrm{~mL}$ ) totalmente separado del resto del estómago, que se une a un segmento proximal de yeyuno de 150$200 \mathrm{~cm}$ de largo, a través de un estoma de $1 \mathrm{~cm}$ de diámetro.

La banda gástrica ajustable es un procedimiento quirúrgico menos invasivo, realizado generalmente por vía laparoscópica, en que una banda de silicona se instala alrededor de la parte superior del estómago dividiéndose éste en dos partes. La porción superior queda con una capacidad de hasta $40 \mathrm{ml}$. Ambas porciones de estómago quedan conectadas por un orificio, cuyo diámetro puede ajustarse rellenando la banda con solución salina isotónica inyectada a través de un reservorio ubicado bajo la piel.

La gastrectomía vertical en manga (Sleeve Gastrectomy en la literatura anglosajona), ha experimentado un uso creciente a nivel mundial en los últimos 5 años. Esta técnica ha sido usada en distintos contextos en obesidad mórbida y en super-obesos ${ }^{42}$, como una primera cirugía en pacientes con alto riesgo quirúrgico antes de un bypass gástrico u otras técnicas más complejas, pero más recientemente se está utilizando como técnica única. Por esta última aplicación se postula que la GVM podría reemplazar a otras cirugías clásicamente restrictivas, tales como la banda gástrica ajustable. La técnica consiste en la sección vertical del estómago, paralelo a la curvatura menor con resección de gran parte del cuerpo y fondo gástrico, dejando un remanente tubular con una capacidad que fluctúa entre 60 y $150 \mathrm{~mL}$, manteniendo el píloro y parte del antro gástrico. $\mathrm{Si}$ bien se han observado resultados alentadores en cuanto a reducción de peso en el corto plazo ${ }^{43-45}$ y mejoría de las comorbilidades asociadas a la obesidad, se desconocen aún los resultados y posibles complicaciones a largo plazo, tales como recuperación del peso perdido ${ }^{46} 0$ deficiencias nutricionales.

Complicaciones de la cirugía en obesidad. Entre los aspectos que preocupan al momento de 
indicar a un paciente una cirugía bariátrica, están la mortalidad y las complicaciones perioperatorias. La mortalidad observada en pacientes sometidos a estas operaciones es menor a $1 \% 30,34$, reportándose $0,1 \%$ en técnicas restrictivas, $0,5 \%$ en bypass gástrico y 1,1\% en derivación biliopancreática, en centros quirúrgicos de excelencia, de acuerdo a los criterios de la Sociedad Americana de Cirugía Bariátrica (Tabla 3$)^{47}$. En una serie nacional de 400 pacientes sometidos a bypass gástrico con gastrectomía, la mortalidad postoperatoria fue de $0,5 \% 39$. Otra serie nacional con análisis de 1.500 pacientes sometidos a bypass gástrico laparoscópico seguidos por 5 años reportó una mortalidad de 0\% (Boza C. et al, Congreso Chileno e Internacional de Cirugía, noviembre de 2008). Las complicaciones perioperatorias después del BPG incluyen tromboembolismo pulmonar, fugas anastomóticas, infección de herida operatoria, sangrado, daño esplénico accidental, hernias incisionales e internas, y obstrucción intestinal. En el estudio $\mathrm{SOS}^{37}$, hubo una frecuencia de $13 \%$ de complicaciones postoperatorias, y en la serie nacional previamente citada ${ }^{39}$ se observaron complicaciones postoperatorias precoces no fatales en $4,8 \%$ de los pacientes, principalmente debido a fuga anastomótica (2,5\%). La anemia (10\%) y la hernia incisional $(10,2 \%)$ fueron las complicaciones tardías más frecuentes en esta serie.

En procedimientos restrictivos puede aparecer náuseas y vómitos en más de $50 \%$ de los pacientes, por ingesta excesiva o demasiado rápida, y a veces por estenosis. En la GVM, además de las potenciales complicaciones de las técnicas restrictivas, se agregan complicaciones propias tales como el sangrado y la filtración a lo largo de la línea de corchetes $^{48}$. En la mayor serie recientemente publicada en 216 obesos mórbidos sometidos a GVM se observaron complicaciones en $6,3 \%$ de los pacientes (filtración en 1,4\% y reoperaciones en $2,8 \%$ ), sin conversiones a BPG ni mortalidad ${ }^{49}$.

Protocolos de tratamiento y seguimiento. Todo paciente que va a ser sometido a cirugía bariátrica debe ser evaluado por un equipo multidisciplinario, con cirujanos digestivos entrenados y experiencia en cirugía bariátrica, médicos con formación y experiencia en asistencia nutricional, nutricionista, psicólogo, psiquiatra y kinesiólogo, y debe ser intervenido en un centro acreditado para dichos procedimientos. Además de la evaluación descrita para todo paciente obeso, el estudio preoperatorio debe incluir exámenes de laboratorio y evaluación de especialistas destinados a reducir el riesgo quirúrgico o anestésico, y de esta forma reducir la mortalidad y las complicaciones postoperatorias $^{33}$. Los centros que realicen estas técnicas quirúrgicas deben contar con el equipamiento apropiado, especialistas interconsultores, protocolos perioperatorios estandarizados y además debieran realizar seguimiento y reporte de resultados a largo plazo. Se recomienda que el seguimiento de todos los pacientes sometidos a cirugía bariátrica sea de por vida.

\section{Tabla 3. C riterios necesarios para formar un centro de excelencia de acuerdo a la Sociedad Americana de Cirugía Bariátrica*}

- Compromiso institucional para realizar un programa educativo

- Más de 125 cirugías bariátricas al año

- Director médico del equipo bariátrico

- Equipo de interconsultores y unidad de cuidados intensivos

- Pabellones quirúrgicos e instrumentos adecuados para pacientes bariátricos

- Cirujanos bariátricos con $51 \%$ de su práctica destinada a cirugía bariátrica

- Protocolos estandarizados de cuidados perioperatorios

- Médico o enfermera de apoyo para educación del paciente bariátrico

- Disponibilidad de grupos de apoyo organizados y supervisados

- Sistema de reporte de resultados y seguimiento a largo plazo

*Adaptado de referencia 47. 


\section{CONCLUSIONES}

La obesidad es una enfermedad crónica, de origen multicausal, caracterizada por un aumento de los depósitos de grasa corporal a niveles de riesgo para la salud. Para su manejo se requiere de un

\section{REFERENCIAS}

1. National Institutes of Health, National Heart Lung and Blood Institute, North American Association for THE StUdy of OBesity. The practical guide for identification, evaluation and treatment of overweight and obesity in adults. Disponible en: http:// www.nhlbi.nih.gov/guidelines/obesity/prctgd_c.pdf

2. WHO. Obesity. Preventing and management the global epidemic. Report of a WHO Consultation on Obesity. Geneva: WHO, 1997.

3. James WP, Astrup A, Finer N, Hilsted J, Kopeiman P, RÖSSNER S ET AL Effect of sibutramine on weight maintenance after weight loss. A randomized trial. STORM study group. Sibutramine trial of obesity reduction and maintenance. Lancet 2000; 356: 2119-25.

4. NARKIEWICZ K. Sibutramine and cardiovascular profile. Int J Obes 2002; 26 (Suppl 4): S38-S41.

5. Vettor R, Serra R, Fabris R, Pagano C, Federspil G. Effect of sibutramine on weight management and metabolic control in type 2 diabetes. A meta-analysis of clinical studies. Diabetes Care 2005; 28: 942-9.

6. Davidson MH, Hauptman J, Digirolamo M, Foreyt JP, Halsted CH, HeBER D et AL. Weight control and risk factor reduction in obese subjects treated for 2 years with orlistat: a randomized controlled trial. JAMA 1999; 281: 235-42.

7. Zhi J, Melia AT, Guerciolni R, Chung J, Kinberg J, HaUPTMAN JB et al. Retrospective population based analysis of the dose response (fecal fat excretion) relationship of orlistat in normal and obese volunteers. Clin Pharmacol Ther 1994; 56: 82-5.

8. KiaIN S. Long-therm pharmacotherapy for obesity. Obes Res 2004; 12: 163S-166S.

9. Torgerson JS, Hauptman J, Boldrin MN, SJöStröm L Xenical in the prevention of diabetes in obese subjects (XENDOS) study: a randomized study of orlistat as adjunct to lifestyle changes for the prevention of type 2 diabetes in obese patients. Diabetes Care 2004; 27: 155-61.

10. Wyatт H. Orlistat and sibutramine in the management of obesity in overweight and the metabolic syndrome, Bray GA and Ryan DH, eds. Springer 2006; 255-79.

11. Sjöström L, Rissanen A, Andersen T, Boldrin M, Golay A, KoppeschaAr HP, KrempF M. Randomized placebocontrolled trial of orlistat for weight loss and equipo multidisciplinario, conformado por médicos especialistas (nutriólogos, endocrinológos), nutricionistas, kinesiólogos y profesores de educación física, equipo de salud mental $\mathrm{y}$, eventualmente, cirujanos con experiencia en técnicas de cirugía bariátrica.

prevention of weight regain in obese patients. European multicentre orlistat study group. Lancet 1998; 352: 167-72.

12. Van Gaal LF, Rissanen AM, Scheen AJ, Ziegler O, ROSSNER S. Effects of the cannabinoid-1 receptor blocker rimonabant on weight reduction and cardiovascular risk factors in overweight patients: 1-year experience from the RIO-Europe study. Lancet 2005; 365: 1389-97.

13. Haddock CK, Poston WS, Dil PL, Foreyt JP, Ericsson M. Pharmacotherapy for obesity: a quantitative analysis of four decades of published randomized clinical trials. Int J Obes 2002; 26: 262-73.

14. Goldstein D, Rampey A, Enas G, Potvin J, Fludzinski L, LEVINE L. Fluoxetine: a randomized clinical trial in the treatment of obesity. Int J Obes 1994; 18: 129-35.

15. Wadden TA, Barlett SJ, Foster GD, Greenstein RA, Wingate BJ, Stunkard AJ et al. Sertraline and relapse prevention training following treatment by very-lowcalorie diet: a controlled clinical trial. Obes Res 1995; 3: 549-57.

16. Knowier WC, Barkett-Connor E, Fowier SE, Hamman RF, LACHIN JM, WaLKer EA, Nathan DM. DPP: Diabetes Prevention Program. Reduction in the incidente of type 2 diabetes with lifestyle intervention or metformine. N Engl J Med 2002; 346: 393-403.

17. Anderson JW, Greenway FL, Fujioka K, Gadde KM, Mc KenNEY J, O'Neil PM. Bupropion SR enhances weight loss: A 48- week double- blind, placebo- controlled trial. Obes Res 2002; 10: 633-41.

18. Bray GA, Holinander P, Kiein S, Kushner R, Levy B, Fitchet M, PerRY BH. A 6-month randomized, placebo-controlled, dose-ranging trial of topiramate for weight loss in obesity. Obes Res 2003; 11: 722-33.

19. Buse JB, Henry RR, Ham J, Kim DD, Fineman MS, Baron AD. Effects of exenatide (exendin-4) on glycemic control over 30 weeks in sulfonilurea-treated patients with type 2 diabetes. Diabetes Care 2004; 27: 2628-35.

20. Maggs D, Shen L, Strobel S, Brown D, Kolterman O, WEYER C. Effect of pramlintide on A1C and body weight in insulin-treated Africans Americans and Hispanics with type 2 diabetes: A pooled post hoc analysis. Metabolism 2003; 52: 1638-42.

21. Heymsfieid SB, Greenberg AS, Fujioka K, Dixon RM, Kushner R, Hunt T et al. Recombinant leptin for weight loss in obese and lean adults: A randomized, 
controlled, dose-escalation trial. JAMA 1999; 282: 1568-75.

22. Smith SR, Prosser WA, Donahue DJ, Morgan ME, ANDERSON CM, Shanahan WR. Lorcaserin (APD356), a selective 5-HT2C agonist, reduces body weight in obese men and women. Obesity 2009; 17: 494-503.

23. BRAY GA, RYan DH. Drug Treatment of the Overweight Patient. Gastroenterology 2007; 132: 2239-52.

24. GLAZER G. Long-term pharmacotherapy of obesity 2000: a review of efficacy and safety. Arch Intern Med 2001; 161: 1814-24.

25. Kernan WN, Viscol CM, Brass LM, Broderick JP, Brott T, Feld mann E et al. Phenylpropanolamine and the risk of hemorrhagic stroke. N Engl J Med 2000; 343: 1826-32.

26. Duшоo AG. Herbal simulation of ephedrine and caffeine in treatment of obesity. Int J Obes 2002; 26: 590-2.

27. Mhurchu CN, Poppitt SD, Mcgill AT, Leahy FE, BenNeTt DA, LN RB ET AL. The effect of dietary supplement, Chitosan, on body weight: a randomized controlled trial in 250 overweight and obese adults. Int J Obes 2004; 28: 1149-56.

28. Díaz E, AguirRe C, Gotteiand M. Efecto de un inhibidor de a-amilasa sobre la reducción de peso de mujeres obesas. Rev Chil Nutr 2004; 31: 306-17.

29. Balsiger BM, Murr MM, Poggio JL, SARr MG. Bariatric Surgery. Med Clin North Am 2000; 84: 477-89.

30. Buchwald H, Avidor Y, Braunwald E, Jensen MD, Pories W, FAHRBACH K ET aL Bariatric Surgery: A Systematic Review and Meta-analysis. JAMA 2004; 292: 1724-37.

31. Maggard MA, Shugarman LR, Suttorp M, Maglione M, Sugerman HJ, Livingston EH et al. Meta-analysis: surgical treatment of obesity. Ann Intern Med 2005; 142: 547-59.

32. NIH Conference: Gastrointestinal surgery for severe obesity. Consensus Development Conference Panel. Ann Intern Med 1991; 115: 956-61.

33. Carrasco F, Kiahssen J, Papapietro K, Reyes E, Rodríguez L, Csendes A Et al. Propuesta y fundamentos para una norma de manejo quirúrgico del paciente obeso. Rev Méd Chile 2005; 133: 699-706.

34. CROоKеS PF. Surgical treatment of morbid obesity. Annu Rev Med 2006; 57: 243-64.

35. ShaH M, SimHa V, Garg A. Long-Term Impact of Bariatric Surgery on Body Weight, Comorbidities, and Nutritional Status. J Clin Endocrinol Metab 2006; 91: 4223-31.
36. Demaria EJ. Bariatric Surgery for Morbid Obesity. N Engl J Med 2007; 356: 2176-83.

37. Sjöström L, Lindroos AK, Peltonen M, Torgerson J, Bouchard C, Carlsson B et al (for the Swedish Obese Subjects Study Scientific Group). Lifestyle, Diabetes, and Cardiovascular Risk Factors 10 Years after Bariatric Surgery. N Engl J Med 2004; 351: 2683-93.

38. Guzmán S, Boza C. Reflexiones sobre la cirugía de la obesidad mórbida. Rev Chil Cirugía 2001; 53: 129-34.

39. Csendes A, Burdiles P, Papapietro K, Díaz JC, Maluenda F, BuRgos A et al. Results of Gastric Bypass Plus Resection of the Distal Excluded Gastric Segment in Patients With Morbid Obesity. J Gastrointest Surg 2005; 9: 121-31.

40. Awad W, Garay A, Martínez C, Oñate V, Turu I, YARMuch J. Experiencia de 10 años con el bypass gástrico. Rev Chil Cirugía 2007; 59: 443-7.

41. Deitel M. Overview of operations for morbid obesity. World J Surg 1998; 22: 913-8.

42. Baltasar A, Serra C, Pérez N, Bou R, Bengochea M, FERRI L Laparoscopic sleeve gastrectomy: a multipurpose bariatric operation. Obes Surg 2005; 15: $1124-28$.

43. Langer FB, Reza-Hoda MA, Bohdjalian A, Felberbauer FX, ZACHerL J, WenZL E ET AL. Sleeve gastrectomy and gastric banding: effects on plasma ghrelin levels. Obes Surg 2005; 15: 1024-9.

44. HaN SM, KIм WW, Он JH. Results of Laparoscopic Sleeve Gastrectomy (LSG) at 1 Year in Morbidly Obese Korean Patients. Obes Surg 2005; 15: 1469-75.

45. Braghetto I, Korn O, Valuadares H, Gutiérrez L, Csendes A, Debandi A et al. Laparoscopic sleeve gastrectomy: surgical technique, indications and clinical results. Obes Surg 2007; 17: 1442-50.

46. Langer FB, Bohdjalian A, Felberbauer FX, Fieischmann E, Reza-Hoda MA, Ludvik B. Does gastric dilatation limit the success of sleeve gastrectomy as a sole operation for morbid obesity? Obes Surg 2006; 16 : 166-71.

47. Nguyen NT, Paya M, Stevens CM, Mavandadi S, Zainabadi $\mathrm{K}, \mathrm{WILSON}$ SE. The relationship between hospital volume and outcome in bariatric surgery at academic medical centers. Ann Surg 2004; 240: 586-93.

48. Melissas J, Koukouraki S, Askoxylakis J, Stathaki M, Daskalakis M, Perisinakis K et al. Sleeve Gastrectomy - A Restrictive Procedure? Obes Surg 2007; 17: 57-62.

49. LeE CM, Cirangle PT, JossarT GH. Vertical gastrectomy for morbid obesity in 216 patients: report of twoyear results. Surg Endosc 2007; 21: 1810-16. 